\section{Nonlinear EMI Simulation of an AM Detector at the System Level}

Sergey L. Loyka

\begin{abstract}
A nonlinear detector simulation technique that can be used together with the discrete technique for a rapid radio receiver electromagnetic compatibility and electromagnetic interference (EMC/EMI) simulation up to the baseband signal processing path at the system level is proposed. The technique presented allows one to simulate an amplitude modulation (AM) detector in the large signal mode as well as in the small signal mode by taking into account the nonlinearity of the detector transfer characteristic. The technique was validated by making comparisons with measurements and PSPICE simulation data.
\end{abstract}

Index Terms-AM detector, electromagnetic interference, system-level simulation.

\section{INTRODUCTION}

Nowadays, the importance of electromagnetic compatibility and electromagnetic interference (EMC/EMI) effects on radio systems is increasing significantly. The extensive growth in radio systems, especially in mobile communication systems, greatly increases the risk of inter and intrasystem EMI [1]. Using appropriate analysis and simulation tools from the initial design stages allows one to optimize the radio system design and performance from an EMC/EMI viewpoint and to reduce significantly the cost of interference prevention.

A radio system can often experience nonlinear behavior and nonlinear phenomena (intermodulation, cross modulation, gain compression/expansion, etc.) and such phenomena have a profound effect on EMC/EMI [1]-[3]. A nonlinear simulation tool must be used in order to carry out EMC/EMI analysis in such a case.

A nonlinear modeling technique (so-called "discrete technique") for numerical EMC/EMI simulation at the system (behavioral) level has been proposed in [4]-[7] (Section II for detail discussion). This technique allows one to carry out rapid numerical EMC/EMI analysis of a complex radio system or subsystem (i.e., receiver, transmitter, etc.) or a set of systems/subsystems over a wide frequency range taking into account nonlinear effects (including spurious responses of a radio receiver). Such an analysis is, for instance, a very important part of EMC/EMI modeling of a mobile communication system [8]-[14].

Unfortunately, the discrete technique cannot be directly applied to a detector simulation because it assumes that the nonlinear element is memoryless and that it operates independently of the linear filter connected to its output (Fig. 1). This is not the case for a detector. For example, the RC circuit at the amplitude modulation (AM)-detector output (Fig. 2) has a profound effect on the diode operation so we cannot analyze the diode and RC-circuit operation separately.

This is an essential limitation on discrete technique possibilities. Due to this limitation, the simulation of a radio receiver can be carried out only as far as the output of the intermediate frequency (IF) path (the detector input) and, correspondingly, base-band signal processing cannot be simulated.

A method which can be used together with the discrete technique so as to simulate an amplitude detector at the system (behavioral) level has been proposed in [15]. However, the nonlinearity of the detector transfer characteristic is not taken into account and therefore

Manuscript received October 28, 1998; revised December 14, 1999.

The author is with the Belorussian State University of Informatics and Radioelectronics, Minsk 220027 Belarus.

Publisher Item Identifier S 0018-9375(00)02173-6.

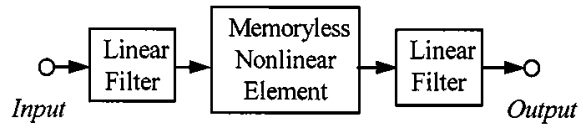

Fig. 1. Representation of a typical radio frequency stage.

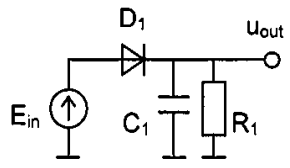

Fig. 2. AM diode detector.

that method can be used in the large signal mode only. Besides, the prediction accuracy of the nonlinear products at the detector output is rather poor in some cases.

Here, the author extends the simulation method given in [15] and presents a system-level AM-detector simulation technique which takes into account the nonlinearity of the detector characteristic and, therefore, can be used both in the small signal and the large signal modes. The method also improves the accuracy of the nonlinear product predictions. The computational time of this method is substantially smaller than that of PSPICE (two to three orders), especially when carrier and modulating frequencies differ significantly. This method also does not require detail schematic knowledge-several system-level detector parameters will suffice.

In Section II, the author gives a brief introduction to the discrete technique. Section III outlines the detector simulation technique given in [15] and its constrains. Section IV gives the improved technique. Section V gives the validation of this technique and in Section VI the frequency and phase detectors simulation are briefly discussed. Finally, Section VII gives conclusions.

\section{OUtLINE OF THE DisCRETE TECHNIQUE}

The main idea of the discrete technique [4]-[6] is that a typical RF stage can be represented as linear filters (LF) and memoryless nonlinear elements (MNE) connected in series (or in parallel). For example, a radio frequency amplifier can be represented as a typical RF stage (so-called "sandwiched nonlinearity"), which employs the linear filter at the input (matching network), the memoryless nonlinear element (transistor) and the linear filter at the output (matching network) [2], as shown in Fig. 1.

The process of signal passage through linear filters is simulated in the frequency domain using the complex transfer factor of the filter

$$
S_{\text {out }}\left(f_{n}\right)=S_{\text {in }}\left(f_{n}\right) \cdot K\left(f_{n}\right)
$$

where

$S_{\text {out }}\left(f_{n}\right) \quad$ signal spectrum at the filter output;

$S_{\text {in }}\left(f_{n}\right) \quad$ signal spectrum at the filter input;

$K\left(f_{n}\right) \quad$ complex transfer factor of the filter;

$f_{n} \quad$ sample frequencies.

A signal passage through a nonlinear memoryless element is simulated in the time domain as

$$
u_{\text {out }}\left(t_{k}\right)=\sum_{i=1}^{I} a_{i} u_{\text {in }}^{i}\left(t_{k}\right)
$$

where

$u_{\text {out }}\left(t_{k}\right) \quad$ instantaneous value of the signal at the MNE output;

$u_{\text {in }}\left(t_{k}\right) \quad$ same for the MNE input; 
$t_{k} \quad$ sample points in time;

$a_{i} \quad$ coefficients of the high-order polynomial which describes the transfer characteristic of the nonlinear element;

I order of the polynomial.

The transition from the time domain to the frequency domain and vice versa is made with the use of the direct and inverse fast Fourier transform (FFT and IFFT)

$$
S=\operatorname{FFT}(u), \quad u=\operatorname{IFFT}(S) .
$$

The determination of the sampling rate, the sample frequency interval, the number of samples as well as a polynomial synthesis technique have been discussed in [5], [7], [16], and [17]. The identification method of nonlinear interference sources has been discussed in [6]. AM-PM conversion can also be modeled with the joint use of the discrete technique and the quadrature modeling technique [10]-[14].

Using the discrete technique, a radio receiver can be simulated over a wide frequency range with a very-high-frequency resolution (up to $10^{6}-10^{7}$ sample frequencies). On a modern PC, analyses can be completed in minutes versus several years for conventional circuit-level simulation.

\section{AM-Detector Simulation at the Large Signal Mode}

Since the primary function of an AM detector is to generate output signal which is proportional to the amplitude of an input signal, it's necessary to calculate the input signal amplitude in order to carry out the simulation. A signal at the input of a radio receiver detector is, as a rule, a narrow-band one, so the Hilbert transform can be used for this purpose. We should note that the requirement for the input signal to be narrowband is dictated not by the Hilbert transform itself which can be applied to a broad-band signal too, but by the RC-circuit present at the detector output. This issue will be discussed further in detail.

For the sampled spectrum which is used in the discrete technique, the Hilbert transform takes the simplest form [18]

$$
S_{n} *=-j \cdot S_{n}
$$

where

$$
\begin{array}{ll}
S_{n}=S\left(f_{n}\right) & \text { sampled spectrum; } \\
S_{n} * & \text { spectrum of the Hilbert conjugate signal; } \\
j & \text { imaginary unit. }
\end{array}
$$

The input signal amplitude can be obtained with the use of the wellknown ratio [18], [19]

$$
A_{k}=\sqrt{u_{k}^{2}+\left(u_{k}^{*}\right)^{2}}
$$

where $u_{k}=u\left(t_{k}\right)$ is the sampled input signal and $u_{k}^{*}$ the Hilbert conjugate signal of $u_{k} . u_{k}^{*}$ is obtained from $S_{n}^{*}$ by means of inverse FFT as

$$
u_{k}^{*}=\operatorname{IFFT}\left(S_{n}^{*}\right) .
$$

In the simplest simulation technique, $A_{k}$ can be used in order to obtain the detector output signal

$$
u_{\text {out }, k}=k_{d} \cdot A_{k}
$$

where $k_{d}$ is the detector transfer factor. This approach works quite well in some practical cases. But, as a detailed consideration shows, there are two constrains for this approach:
1) the bandwidth of the input signal must be smaller than the cutoff frequency of a low-pass filter (RC circuit) at the detector output

$$
\Delta f_{\text {in }}<F_{\text {cut }}
$$

(in some cases this constrain can be relaxed, as shown later);

2) the input signal must be large enough so that the detector operates in the large signal mode

$$
A_{k}>A_{\text {min }}
$$

where $A_{\min }$ is a threshold level which is determined by the volt-ampere characteristic of the nonlinear device used in the detector (for a diode detector $A_{\min } \approx 0.2 \div 0.7 \mathrm{~V}$ and for a transistor $A_{\min } \approx 50 \div 100 \mathrm{mV}$ )

The second constrain is rather obvious - the output signal in an AM detector is proportional to the input signal amplitude ( $k_{d}$ is a constant) only when operating in the large signal mode. In the small signal mode $k_{d}$ is a function of $A_{k}$ and the spectral content of the output signal is much richer than that of $A_{k}$. This technique doesn't differentiate between the small and large signal modes since the Hilbert transform is linearly related to the input signal amplitude as

$$
u_{k}^{*}\left(c \cdot u_{k}\right)=c \cdot u_{k}^{*}\left(u_{k}\right)
$$

where $c$ is a constant and, correspondingly, $A_{k}\left(c \cdot u_{k}\right)=c \cdot A_{k}\left(u_{k}\right)$. For this reason, this technique cannot predict harmonics of the modulating signal, which always are present at the detector output (fortunately, for most of practical detectors, the harmonic levels are rather low and may be disregarded in EMC problems).

To discuss the first constrain in detail, let us consider the detector shown in Fig. 2.

If the bandwidth of the input signal $E_{\mathrm{in}}$ (which contains interference as well as a required signal) is smaller than the cutoff frequency of the RC-circuit at the detector output, the output signal $u_{\text {out }}$ repeats the amplitude of the input signal at the same moment of time and thus the detector is said to be memoryless. If not, the output signal doesn't repeat the amplitude of the input signal and depends on its levels at the preceding moments of time and the detector is said to have memory. To determine the boundary between these two modes, lets consider the input signal shown in Fig. 3. This signal consists of the required AM signal $\left(f_{c}, f_{c}+F_{m}\right.$ and $\left.f_{c}-F_{m}\right)$ and the interference signal $\left(f_{\text {int }}\right)$. The required signal bandwidth is always smaller than the cutoff frequency (the design constrain). The interaction between the interference and the required signal will result in a beat. So we must consider the difference between the interference frequency and the required signal frequencies (the beat frequencies). Taking into account that the side frequencies levels are, as a rule, smaller than that of the carrier and for the sake of simplicity, we shall consider further the beat between the carrier and the interference (beat between the side frequencies and the interference can be considered in a similar way). Then the signal bandwidth is equal to the beat frequency

$$
\Delta f_{\text {in }}=f_{\text {beat }}=f_{\text {int }}-f_{c}
$$

where $f_{\text {beat }}$ is the beat frequency. If condition (8) is true, then the output signal repeats the input signal amplitude and the technique works quite well. Otherwise, the output signal doesn't repeat the input signal amplitude because capacitor $C_{1}$ has not managed to discharge with the beat frequency: the rate of the capacitor discharge $v_{C}$ is smaller than the rate of the change of the beat signal amplitude $v_{\text {beat }}$. Let's assume for simplicity that $u_{\text {int }}<u_{c}$, where $u_{\text {int }}$ is the interference level and $u_{c}$ is the required signal level without modulation (the opposite case can be considered in a similar manner). Then, taking 


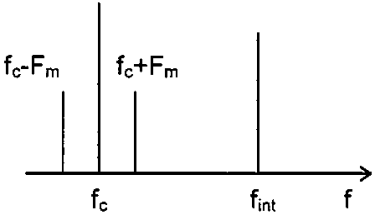

Fig. 3. Spectrum of the input signal: $f_{c}$-carrier frequency, $F_{m}$-modulating frequency, and $f_{\text {int }}$-interference frequency.

into account the exponential law for the capacitor discharge, we obtain the following assessment for $v_{C}$ :

$$
v_{C} \approx \frac{u_{c}}{\tau}
$$

where $\tau=R_{1} C_{1}$ is the RC-circuit constant. In a similar way we obtain the following assessment for the average value of $v_{\text {beat }}$ :

$$
v_{\text {beat }} \approx \frac{4 u_{\text {int }}}{T_{\text {beat }}}
$$

where $T_{\text {beat }}=1 / f_{\text {beat }}=1 / \Delta f_{\text {in }}$ is the beat period, since the beat amplitude equals the interference amplitude. Our simulation technique will predict the correct output signal if $v_{C}>v_{\text {beat }}$, or, using (12) and (13)

$$
u_{\text {int }}<\frac{u_{c}}{4 \tau \cdot f_{\text {beat }}} .
$$

From this condition we can conclude the following: if the interference level is rather low, then the output interference level (at the beat frequency) is not affected by the RC-circuit even if the beat frequency is larger than the cutoff frequency of the RC-circuit (this conclusion is also confirmed by the PSPICE simulation and by measurements).

Thus, it is absolutely unacceptable to model the AM detector as the series connection of a nonlinear element (or a frequency transformer) and a low-pass filter, as it has been proposed by some authors. Physically it can be explained as follows: the capacitor is discharged through the resistor $R_{1}$ and is charged through the direct diode resistance which is much smaller than $R_{1}$, so the discharge time constant and the charge time constant are quite different.

From the practical viewpoint, spectral components of the detector input signal, which lie outside of the IF path bandwidth, will be strongly attenuated by the IF path, so that condition (14) will be fulfilled. If, nevertheless, it is not, it means that these spectral components have very large level at the receiver front-end stages and the receiver is completely blocked.

If condition (14) is not true, then the output interference signal will be attenuated by the RC circuit. But the attenuation factor will be smaller then the transfer factor of the RC circuit. Thus, the optimal decision is to ignore completely the RC circuit during the simulation.

We should note that if an additional low-pass filter is connected to the detector output (as on Fig. 4), then this filter must be taken into account (since the capacitor $C_{2}$ charge and discharge time constants are the same) as

$$
\bar{S}_{\text {out }}=S_{\text {out }} \cdot K(f)
$$

where $\bar{S}_{\text {out }}$ is the spectrum at the filter output and $K(f)$ is the complex transfer factor of the filter.

Let's consider the sampling rate, the sample frequency interval and the number of samples. These quantities are determined using methods similar to those in the discrete technique [4],[5]. The maximum sample frequency $f_{n, \max }$ must be higher than the highest input spectrum frequency $f_{\text {in, } \max }$ with some margin, which can be expressed as

$$
f_{n, \max }=k f_{\text {in, } \max }
$$

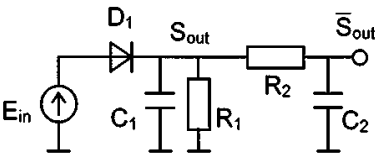

Fig. 4. AM diode detector with a low-pass filter.

where $k$ is a margin factor $(k=2 \cdots 10)$. The sample frequency interval $\Delta f_{n}$ must be lower with some margin than the lowest input beat (or modulating) frequency $f_{\text {beat, min }}$, which should be modeled, which can be expressed as

$$
\Delta f_{n}=k_{1} f_{\text {beat }, \text { min }}
$$

where $k_{1}$ is a margin factor $\left(k_{1}=0.1 \cdots 0.5\right)$. Using (16) and (17), the number of samples in the time domain can be expressed as

$$
N=2 \frac{f_{n, \max }}{f_{\text {beat }, \min }} .
$$

We must round off this number to a power of two (in order to use FFT) as

$$
\bar{N}=2^{m}, m=\left[\log _{2} N\right]+1
$$

where $[*]$ is the whole part. Further we recalculate $\Delta f_{n}$ for constant $f_{n, \max }$ (or $f_{n, \max }$ for constant $\Delta f_{n}$ ) using (16)-(18).

\section{NONLINEAR AM-DETECTOR SIMULATION}

In order to simulate an AM-detector in the small signal mode and to predict accurately the levels of nonlinear products at its output, its necessary to take into account the nonlinearity of the detector characteristic. When the output impedance of a detector is much smaller than the load impedance, the following formula for $k_{d}$ [20] can be used

$$
k_{d}=\frac{\ln I_{0}\left(\overline{A_{k}}\right)}{\overline{A_{k}}}
$$

where $\bar{A}_{k}=A_{k} / \varphi_{t e}$ is the normalized amplitude, $\varphi_{t e}=\alpha \varphi_{t}$ is the effective thermal voltage, $\alpha$ is a correction factor (it depends on the type of a detector element, $\alpha=1 \cdots 3$ ), $\varphi_{t}$ is the thermal voltage ( $\varphi_{t} \approx 25 \mathrm{mV}$ for the room temperature), and $I_{0}$ is the zeroth order modified Bessel function of the first kind.

Taking into account all considerations given above, we can present the AM-detector simulation scheme as shown in Fig. 5.

As a practical experience shows, this technique works quite well for a transistor AM detector (the output impedance of such a detector is much smaller than the load impedance), but fails to work for a diode AM detector since the output impedance of such a detector in the small signal mode is much larger than the load impedance. Thus, in this case we must correct (20) to account for the detector output impedance. To accomplish this, the method given in [20] and [21] is used. The basic equation for the detector transfer factor is [20]

$$
\ln (Y+1)+Y \cdot \beta=\ln I_{0}\left(\overline{A_{k}}\right)
$$

where $Y=u_{\text {out }, k} / R_{1} \cdot I_{s}, R_{1}$ is the load resistance (see Figs. 2 and 4) and $I_{s}$ is the diode saturation current, $\beta=I_{s} R_{1} / \varphi_{t e}$. Using (21), the detector transfer factor can be expressed as follows:

$$
k_{d}=\frac{u_{\mathrm{out}, k}}{A_{k}}=\frac{1}{\overline{A_{k}}} \ln \left(\frac{I_{0}\left(\overline{A_{k}}\right)}{Y+1}\right) .
$$

To find $k_{d}$, we must solve (21) for $Y$. Unfortunately, this equation is transcendental for $Y$ and it has no analytical solution in general. It is unsuitable to use a numerical technique to solve the equation since the 


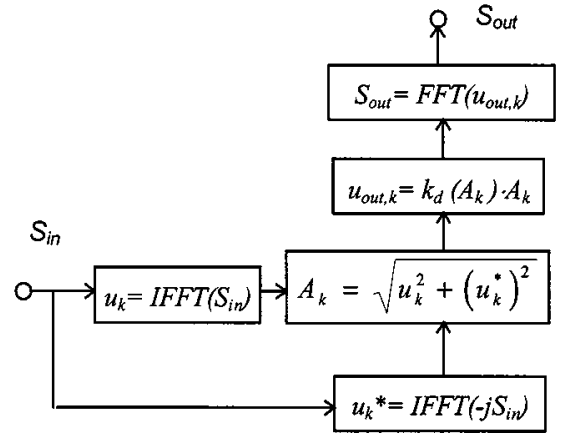

Fig. 5. Nonlinear AM-detector simulation scheme: $S_{\text {in }}$-input signal spectrum and $S_{\text {out }}$-output signal spectrum.

simulation time increases substantially. To find a solution, let us use the perturbation theory, i.e., to present $Y$ in the form

$$
Y=Y_{0}+\Delta Y
$$

where $Y_{0}$ is the solution of $(21)$ for $\beta=0$,

$$
Y_{0}=I_{0}\left(\overline{A_{k}}\right)-1 \text {, }
$$

and $\Delta Y$ is an addition to $Y_{0}$ when $\beta \neq 0$. Using (23), we rewrite (21) in the form

$$
\ln \left(Y_{0}+\Delta Y+1\right)+\left(Y_{0}+\Delta Y\right) \cdot \beta=\ln I_{0}\left(\overline{A_{k}}\right) .
$$

Using (21a) and (22)-(24), after some manipulations we obtain the following equation for $k_{d}$ :

$$
k_{d}=\frac{1}{\overline{A_{k}}} \cdot \ln \left(\frac{1+\beta \cdot I_{0}\left(\overline{A_{k}}\right)}{1+\beta}\right) .
$$

This equation generalizes (20) for the case of large output detector impedance (when $\beta \leq 1$; a typical value of $\beta$ for a diode detector is $\beta=0.01 \cdots 0.1$ ). Fortunately, (25) reduces to (20) when the output impedance is small ( $\beta \gg 1$ ), so it works for large values of $\beta$ too (we should note that, as a rule, perturbation series work well for a small value of an expansion parameter and its rather difficult to obtain a good approximation for large value of the expansion parameter).

Thus, the diode AM-detector simulation can be carried out as on Fig. 5 but $k_{d}$ is calculated using (25) rather than (20).

\section{TECHNIQUE VALIDATION}

In order to validate the technique proposed, extensive circuit-level simulations of diode and transistor AM-detectors and measurements have been performed. The circuit-level simulations were performed using the well-known simulation tool PSPICE [22]. The signal shown in Fig. 3 was used as the input during the simulations and measurements.

Fig. 6 shows a measurement block diagram. It includes an AM signal generator, an interfering signal generator, a combiner, an AM detector, a buffer stage (it was used to match the detector output impedance with the spectrum analyzer input impedance), and a spectrum analyzer. First, the measurement setup itself (without the detector) was tested to be sure that it did not generate nonlinear products. Then, the output detector signal was measured.

Some results of the simulation and measurements and the comparison with the technique proposed for the transistor detector are presented in Figs. 7 and 8.

For Fig. 7, the interference frequency is within the required signal bandwidth (parameters of the input signal: $f_{c}=1 \mathrm{MHz}$,
$F_{m}=10 \mathrm{kHz}$, modulation index $m=0.3, u_{c}=100 \mathrm{mV}$, $u_{\text {int }}=300 \mathrm{mV}, f_{\text {int }}=1015 \mathrm{kHz}$ ). As it can be seen from this figure, the proposed technique agrees well with PSPICE predictions and with measurements for levels not smaller than $-30 \cdots-40 \mathrm{~dB}$ relative to the maximum. All effects known from the theory (signal compression, beat generation at the frequencies $f_{\text {int }}-f_{c}, f_{\text {int }}-f_{c}-F_{m}$, $f_{\text {int }}-f_{c}+F_{m}$, etc.) are predicted quite well. For example, the output detector signal at $10 \mathrm{kHz}$ (modulating frequency) would be $30 \mathrm{mV}$ $\left(\approx m \cdot u_{c}\right.$ in the large signal mode) without the interference signal, and in our case it is $\approx 5 \mathrm{mV}$. During the computations, we found that the proposed technique is approximately a hundred times faster than PSPICE.

For Fig. 8, the interference frequency lies outside of the required signal bandwidth (parameters of the input signal: $f_{c}=1 \mathrm{MHz}, F_{m}=$ $\left.10 \mathrm{kHz}, m=0.3, u_{c}=100 \mathrm{mV}, u_{\text {int }}=100 \mathrm{mV}, f_{\text {int }}=1100 \mathrm{kHz}\right)$. As it can be seen from this figure, the largest beat level at the frequency $f_{\text {int }}-f_{c}=100 \mathrm{kHz}$ is predicted quite well. It proves our conclusion that the RC circuit must be discarded during the simulation (for the present case, $\left(f_{\text {int }}-f_{c}\right) / F_{\text {cut }} \approx 3$ so if the RC circuit had operated as an usual low-pass filter, threefold attenuation would have been expected for this beat frequency, which is not observed in reality).

Some results of the simulation and measurements and the comparison with the technique proposed for the diode detector (see Fig. 2) are presented on Figs. 9 and 10. Fig. 9 shows the diode detector transfer characteristic (output level at the modulating frequency versus carrier amplitude at the input). Parameters of the input signal are as follows: $f_{c}=1 \mathrm{MHz}, F_{m}=10 \mathrm{kHz}, m=0.3$. As one can see from this figure, the proposed technique predicts the transfer characteristic quite accurately.

Fig. 10 shows spectrum at the detector output for the input signal as shown in Fig. 3 (parameters of the input signal: $f_{c}=1 \mathrm{MHz}, F_{m}=$ $\left.10 \mathrm{kHz}, m=0.3, u_{c}=300 \mathrm{mV}, u_{\text {int }}=1 \mathrm{~V}, f_{\text {int }}=1015 \mathrm{kHz}\right)$. As we can see from this figure, the technique proposed predicts the spectral component levels accurately down to $-30 \cdots-40 \mathrm{~dB}$ relative to the maximum.

We should note the following.

- Spectral levels at the diode AM-detector output in the small signal mode depend substantially on the diode saturation current (i.e., $\beta$ ) which in turn reveals large sample-to-sample fabrication dispersion. Thus, the prediction accuracy is not very good in this case (the typical value is about $10 \mathrm{~dB}$ ) and the use of a circuit-level simulator can not improve the situation for this reason. Small spectral components in the large signal mode can not be predicted with high accuracy as well. The Monte Carlo simulation method could be a solution to this problem.

- If the figure of merit is the signal/interference ratio or similar, then the maximum level in the interference spectrum gives the main contribution. This level is predicted by the technique proposed quite well.

- When the values of $I_{s}$ and $\alpha$ are extracted from the measurement of the diode volt-ampere characteristic (as in PSPICE, for instance), only the small-signal part of this characteristic should be taken into account in order to achieve good accuracy for these values. The large signal part of this characteristic can reduce the accuracy substantially.

\section{FM/PM DETECTOR SIMULATION}

A similar approach can be used in order to simulate an FM or PM detector. Using the Hilbert Transform, we find instant angular frequency of the detector input signal [18] as

$$
\omega_{k}=\frac{u_{k}^{*} \cdot u_{k-1}-u_{k-1}^{*} \cdot u_{k}}{\Delta t \cdot A_{k}^{2}}
$$




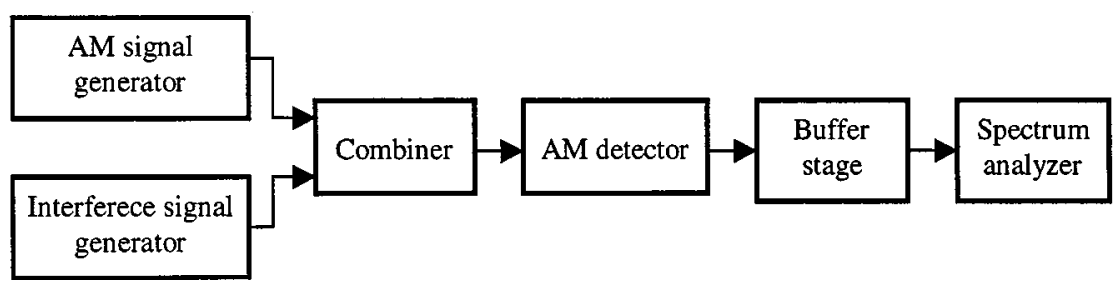

Fig. 6. Measurement block diagram.

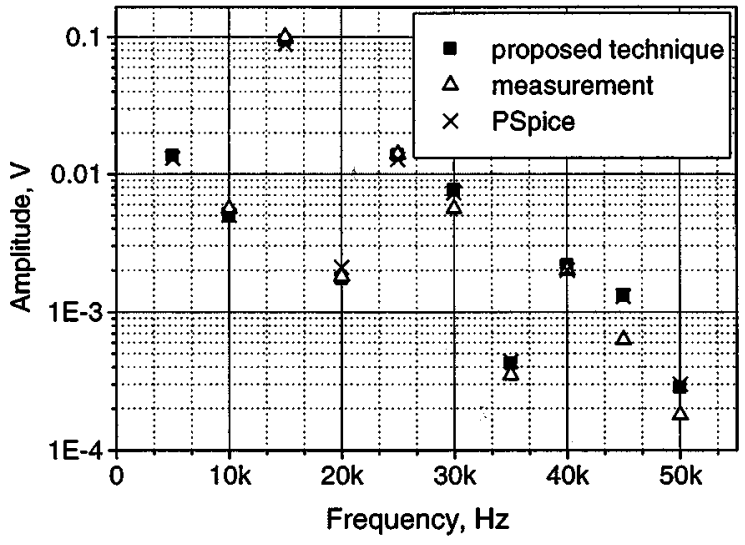

Fig. 7. Spectrum at the transistor AM-detector output (input signal—as shown on Fig. 3). The interference lies within the required bandwidth.

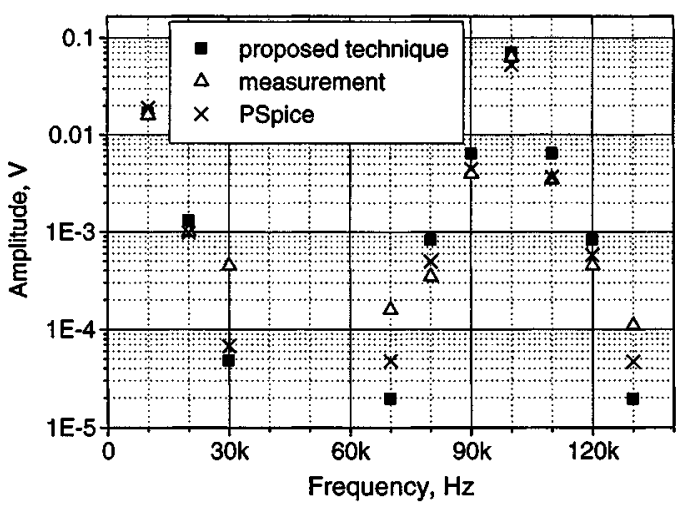

Fig. 8. Spectrum at the detector output (input signal—as shown on Fig. 3). The interference lies outside the required bandwidth.

where $\Delta t$ is the time sample interval. Output signal of an FM detector is proportional to the difference between the instant frequency and the detector resonant frequency $\omega_{0}$ and is expressed as

$$
u_{\text {out }, k} \approx k_{d}\left(\omega_{k}-\omega_{0}\right) .
$$

This equation is valid for the linear part of the detector input-output characteristic when

$$
\left|\omega_{k}-\omega_{0}\right| \leq \Delta \omega
$$

where $\Delta \omega$ is the linear part width, and for a sufficiently large input signal when its amplitude is constant (due to the limiter which is connected in front of the detector). This can be expressed as

$$
A_{\text {lim, in }} \geq A_{t h,, \text { in }}
$$

where $A_{\lim \text {, in }}$ is a signal amplitude at the limiter input and $A_{t h}$, in its threshold level (the saturation level). In other cases, this equation

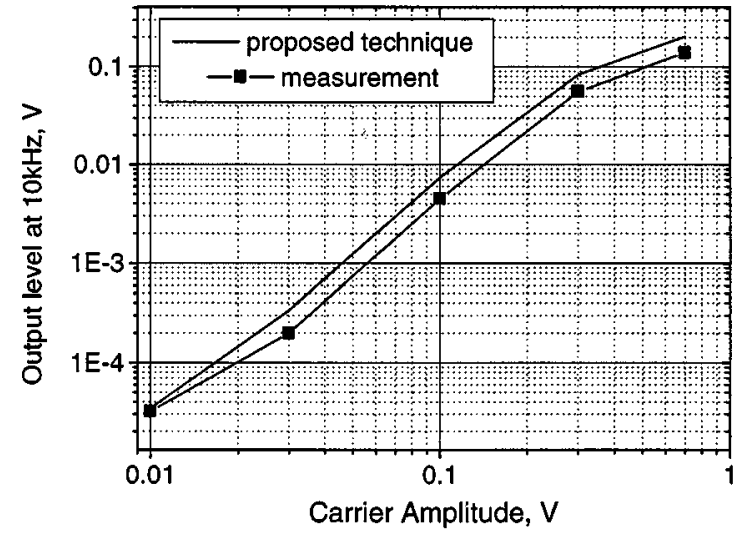

Fig. 9. The diode AM-detector transfer characteristic.

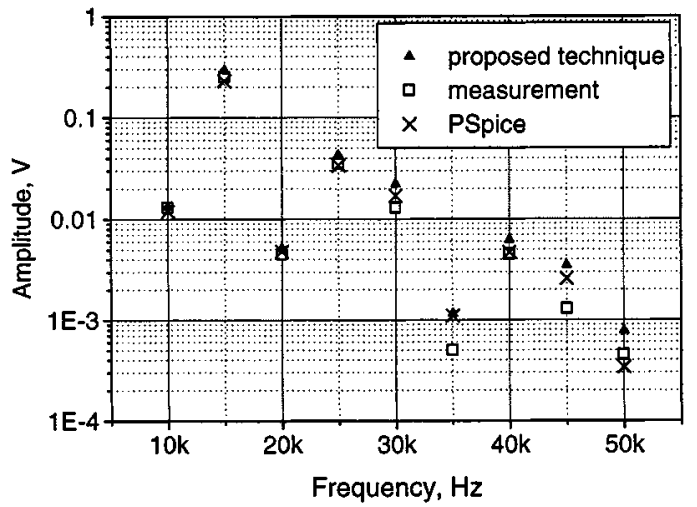

Fig. 10. Spectrum at the diode AM-detector output (input signal—as shown on Fig. 3). The interference lies within the required bandwidth.

should be generalized to take into account the nonlinearity of the detector characteristic and its dependence on the input signal amplitude

$$
k_{d}=k_{d}\left(\omega_{k}-\omega_{0}, A_{k}\right) \text {. }
$$

Appropriate approximations for the dependence of $k_{d}$ on $\omega_{k}-\omega_{0}$ can be found in [20]. The dependence of $k_{d}$ on $A_{k}$ can be approximated by

$$
k_{d} \approx c \cdot A_{k}, \quad c \text { - constant }
$$

for an FM detector with tuned-off circuits or similar and by

$$
k_{d} \approx c \cdot A_{k}^{2}, \quad c-\text { constant }
$$

for an FM detector with a multiplier.

As practical experience shows, this simulation technique predicts the required signal compression and the threshold effect quite well. Predicted interference levels are smaller than in reality since the nonlinearity of the detector amplitude characteristic is not taken into account. 


\section{CONCLUSION}

A nonlinear technique of system-level detector simulation has been proposed in the paper. This technique can be used for the EMC/EMI simulation of a complex radio system together with the discrete technique when the use of a circuit-level simulator is not possible in view of limited computer resources or when the exact circuit parameters are not known. The computational efficiency of the technique proposed is two to three orders higher than that of PSPICE, especially when carrier and modulating frequencies differ significantly.

In general, we can conclude that the technique proposed predicts output spectral components of an AM detector, which are not smaller than $-30 \mathrm{~dB}$ relative to the maximum level, quite accurately. Phase and frequency detectors can be simulated using an approach similar to that applied to the AM-detector simulation.

\section{REFERENCES}

[1] J. Gavan, "Principles of radio system and relevant EMI environmental effects, analysis and computation," in Handbook on Electromagnetic Compatibility, R. Perez, Ed. New York: Academic, 1995.

[2] D. D. Weiner, "Nonlinear interference effects in EMC," in Supplement Proc. 10th Int. Zurich Symp. Electromagn. Compat., Zurich, Mar. 1993, pp. 114-127.

[3] J. Gavan and E. Joffe, "Nonlinear radio mutual interference main effect analysis and mitigation methods," in Proc. IEEE Int. Symp. Electromagn. Compat., Denver, CO, Aug. 1998, pp. 74-79.

[4] V. I. Mordachev, "Express analysis of electromagnetic compatibility of radio electronic equipment with the use of the discrete models of interference and fast Fourier transform," in Proc. IX Int. Wroclaw Symp. Electromagn. Compat., Wroclaw, Poland, 1988, pp. 565-570.

[5] S. L. Loyka and V. I Mordachev, "Computer-aided nonlinear simulation at the system level," in Proc. 5th Int. Conf. EMC/EMI (INCEMIC'97), Hyderabad, India, Dec. 1997, pp. 93-98.

[6] - "Identification of nonlinear interference sources with the use of the discrete technique," in IEEE Int. Symp. Electromagn. Compat., Denver, CO, Aug. 1998, pp. 882-887.
[7] I. D. Cheremisinov, S. L. Loyka, and V. I Mordachev, "Synthesis of the polynomial model of nonlinear elements based on intermodulation dynamic ranges," in Proc. 3rd Int. Conf. Telecommun. Modern Satellite, Cable, Broadcasting Services (TELSIKS'97), Nis, Yugoslavia, Oct. 1997, pp. 519-522.

[8] S. W. Chen, W. Panton, and R. Gilmore, "Effects of nonlinear distortion on CDMA communication systems," IEEE Trans. Microwave Theory Tech., vol. 44, pp. 2743-2750, Dec. 1996.

[9] B. Gallagher, "Estimating and measuring C/I in a GSM wireless local loop receiver," Microwave J., vol. 40, no. 10, pp. 70-83, Oct. 1997.

[10] J. Staudinger, "Applying the quadrature modeling technique to wireless power amplifiers," Microwave J., vol. 40, no. 11, pp. 66-86, Nov. 1997.

[11] S. L. Loyka, "The influence of electromagnetic environment on operation of active array antennas: Analysis and simulation techniques," IEEE Antennas Propagat. Mag., vol. 41, pp. 23-39, Dec. 1999, to be published.

[12] _ - "Numerical simulation of nonlinear interference in radio systems," in 26th URSI Gen. Assembly, Toronto, Canada, Aug. 1999, Invited Paper.

[13] - New behavioral level simulation technique for RF/microwave applications, Int. J. RF Microwave CAE, to be published.

[14] F. Amoroso and R. A. Monzingo, "Spectral sidelobe regrowth in saturating amplifiers," Appl. Microwave Wireless, vol. 10, no. 2, pp. 36-42, Mar. 1998.

[15] S. L. Loyka, "Detector simulation with the use of the discrete technique," in Proc. 14th Int. Wroclaw Symp. Electromagn. Compat., Wroclaw, Poland, June 1998, pp. 311-315.

[16] S. L. Loyka and V. I. Mordachev, "A polynomial approximation of an ideal limiter characteristic for a functional simulating of receiving device" (in Russian), Electrosviaz, (Telecommun. Radio Eng.), vol. N7, pp. 32-33, 1995.

[17] V. I. Mordachev and I. D. Cheremisinov, "Polynomial models of amplitude characteristic of limiting elements for the discrete analysis of EMC of radioelectronic equipment" (in Russian), Izvestia Vuzov. Radioelectronica (Radioelectron. Commun. Syst.), vol. 39, pp. 38-47, Aug. 1996.

[18] S. I. Baskakov, Radio Engineering Circuits Signals (in Russian), V. Shkola, Ed. Moscow, Russia, 1988

[19] V. N. Il'in, Ed., Computer-Aided Circuit Design (in Russian). Moscow, Russia: Radio I Cvjaz, 1987

[20] V. I. Siforov, Ed., Radio Receivers (in Russian). Moscow, Russia: Sovietskoe Radio, 1974

[21] S. L. Loyka, "Simple formula for AM-detector transfer factor," Electron. Lett., vol. 35, no. 2, pp. 126-127, Jan. 1999.

[22] MicroSim PSPICE \& Basics: User's Guide, MicroSim Corp., Irvine, CA, 1996. 